\title{
Medication use in patients with chronic rhinosinusitis in Germany - a large retrospective patient-based study*
}

\author{
Jonas Jae-Hyun Park' , David Ulrich Seidel2, Claus Bachert ${ }^{3}$, Stefan Dazert', \\ Karel Kostev ${ }^{4}$ \\ ' Department of Otorhinolaryngology and Head and Neck Surgery, Ruhr University Bochum, St. Elisabeth-Hospital, Bochum, \\ Germany \\ ${ }^{2}$ Department of Otorhinolaryngology, Head and Neck Surgery, Solingen Municipal Hospital, Solingen, Germany \\ 3 Upper Airways Research Laboratory and ENT-Department, Ghent University Hospital, Ghent, Belgium \\ ${ }^{4}$ Epidemiology, IQVIA, Frankfurt, Germany
}

Rhinology 57: 2, 94 - 100, 2019

https://doi.org/10.4193/Rhin18.055

*Received for publication:

March 14, 2018

Accepted: July 10, 2018

Background: The aim of the present study was to provide an insight into medical treatment practices among patients with chronic rhinosinusitis (CRS) in Germany. An investigation of ICD codes and ATC classes of CRS patients in general and otolaryngology offices in Germany should reveal the prevalent treatment behaviors of German physicians.

Methods: The present study used data from the Disease Analyzer database (IQVIA). The study sample included patients from 940 general (GP) and 106 otolaryngology (ENT) practices who were coded as having 'chronic sinusitis' (ICD-10: J32) or 'nasal polyps' (ICD-10: J33) in 2015 (index date). The primary outcome measures were the number of patients with these diagnoses per practice as well as the proportion of patients with prescriptions for topical corticosteroids, systemic corticosteroids, antibiotics, antihistamines, and local decongestants within 365 days after the first diagnosis.

Results: This retrospective study included 26,768 patients with coding for 'chronic sinusitis' (ICD-10: J32) and 516 patients for'nasal polyps' (ICD-10: J33) in 940 GP practices and 19,826 patients with coding for 'chronic sinusitis' (ICD-10: J32) and 1,773 patients for 'nasal polyps' (ICD-10: J33) in 106 ENT practices.

In patients coded as having 'chronic sinusitis' (ICD-10: J32), topical corticosteroids were prescribed at a low rate (GP: 12.3\%, ENT: 34.3\%). In patients coded as having 'nasal polyps' (ICD-10: J33), topical corticosteroid usage was higher in GP practices (27.3\%) and in ENT practices (71.2\%).

Conclusions: Topical corticosteroid usage in CRS patients in GP practices in Germany is as low as in other Western countries. Increased usage of topical corticosteroids in CRS patients with polyposis should be encouraged in GP and ENT practices.

Key words: chronic rhinosinusitis, nasal polyps, medication

\section{Introduction}

With a prevalence of approximately $10 \%$, chronic rhinosinusitis (CRS) is one of the most common diseases in Western countries (1). CRS is often treated medically for a prolonged period of time. Corticosteroids have been a major component in such medical therapy regimes. Topical steroids are considered as part of the standard treatment, since they have been shown to have a superior effect on symptoms compared to placebo ${ }^{(2)}$. In acute exacerbations, recurrent disease, and clinical episodes refractory to local medication, systemic steroids are commonly administered on a temporary basis, although their beneficial effects as a single therapy have been subject to debate ${ }^{(3)}$. To date, corticosteroids have been incorporated into various practical guidelines. The "European position paper on rhinosinusitis and nasal polyps" (4), the "Canadian clinical practice guidelines for acute and chronic rhinosinusitis" (5), and the "Clinical practice guideline (update): adult sinusitis" (6) recommend them for the treatment of CRS.

Furthermore, systemic antibiotics have been another major component of general CRS treatment. Antibiotics are primarily prescribed in patients exhibiting acute exacerbations (with or without bacterial superinfection), in order to improve sudden 
worsening of symptoms. Although long-term usage of macrolide antibiotics has been studied, results are not consistent ${ }^{(7,8)}$. Use of antihistamines in CRS patients has been reported ${ }^{(9)}$ but is not recommended according to the EPOS ${ }^{(4)}$. As of late, data has been scarce in terms of current medication usage and prescription habits for CRS treatment. Interestingly, Canadian data showed restrained application of intranasal corticosteroids (ICS). Only $20 \%$ of patients who did not undergo surgical treatment within 3 years received ICS ${ }^{(10)}$. Furthermore, investigations in the United Kingdom (UK) also found similarly low application percentages of ICS. Among patients with chronic rhinosinusitis without polyposis (CRSsNP) only $12.1 \%$ and among patients with chronic rhinosinusitis with polyposis (CRSwNP) only $18.7 \%$ used ICS (11).

Until now, there have been no epidemiological studies regarding the medical treatment behavior for CRS in Germany. Data retrieved from larger population groups is rarely reported in general and otolaryngology offices. CRS is a highly prevalent disease which greatly affects national health costs. Therefore it is important to be aware of prescription patterns in the outpatient setting. The aim of the present study was to provide an insight into medical treatment practices among patients with CRS in Germany. An investigation of ICD codes and ATC classes of CRS patients in general and otolaryngology offices in Germany should reveal the prevalent treatment behaviors of German physicians.

\section{Materials and methods}

The present study used data from the Disease Analyzer database (IQVIA), which compiles drug prescriptions, diagnoses, and basic medical and demographic data obtained directly and in anonymous format from computer systems used in physicians' practices. Diagnoses (ICD-10), prescriptions (Anatomical Therapeutic Chemical [ATC] Classification System), and the quality of reported data are monitored by IQVIA based on a number of criteria (e.g., completeness of documentation, linkage between diagnoses and prescriptions, etc.). Due to the retrospective nature of anonymized data from primary and secondary care practices throughout Germany, no specific ethical consent was obtained ${ }^{(12)}$. The database has already been used in several studies focusing on ENT diseases ${ }^{(13,14)}$.

The study sample included patients from 940 general (GP) and 106 otolaryngology (ENT) practices who had been diagnosed with 'chronic sinusitis' (ICD-10: J32) or 'nasal polyps' (ICD-10: J33) between January 2015 and December 2015 (index date). The primary outcome measures were the number of patients with these diagnoses per practice as well as the proportion of patients with prescriptions for topical corticosteroids (EphRA ATC: R01A1, R01A3), systemic corticosteroids (H02), antibiotics (J01), antihistamines (R06A), and local decongestants (R01AY7) within 365 days after the first diagnosis of 'chronic sinusitis' (ICD-

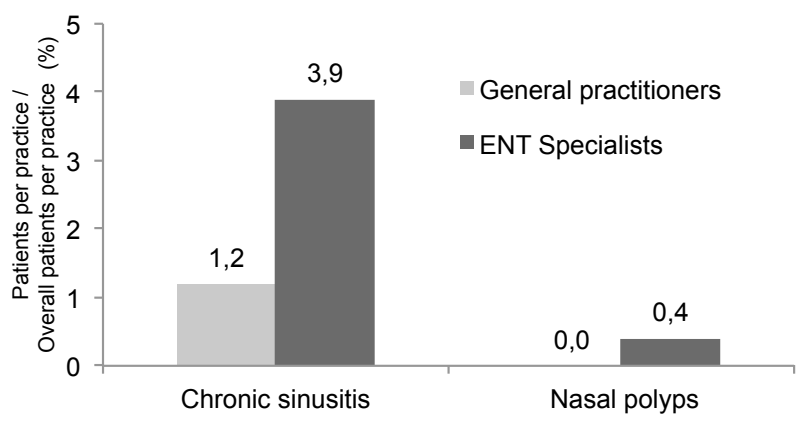

Figure 1. Patients with chronic sinus diseases in relation to the overall number of patients per practice.

10: J32) or 'nasal polyps' (ICD-10: J33). The present study contains only descriptive analyses, and no specific hypotheses were tested. Analyses were carried out using SAS version 9.4.

\section{Results}

This retrospective study included 26,768 patients coded as having 'chronic sinusitis' (ICD-10: J32) and 516 patients coded as having 'nasal polyps' (ICD-10: J33) in 940 GP practices as well as 19,826 patients coded as having 'chronic sinusitis' (ICD-10: J32) and 1,773 patients coded as having 'nasal polyps' (ICD-10: J33) in 106 ENT practices. The mean age of 'chronic sinusitis' patients (ICD-10: J32) was 39.2 years (SD: 17.1) in GP practices and 42.5 (SD: 18.6) in ENT practices; the mean ages of 'nasal polyp' patients (ICD-10: J33) were 43.0 (SD: 21.2) and 48.9 (SD: 18.3), respectively, for the two practice types. $42.4 \%$ of 'chronic sinusitis' patients (ICD-10: J32) treated by GPs and $43.2 \%$ treated by ENTs as well as $55.4 \%$ of 'nasal polyp' patients (ICD-10: J33) treated by GPs and $61.4 \%$ treated by ENTs were male.

The number of patients per practice was higher in ENT than in GP practices (Figures 1, 2). Data showing the number of chronic sinusitis patients per German practice are listed in Table 1. The prescription frequencies of topical corticosteroids, systemic corticosteroids, antibiotics, antihistamines, and local decongestants in patients coded as having 'chronic sinusitis' (ICD-10: J32) are shown in Figure 3. GPs prescribed these medications to $12.3 \%$, $4.1 \%, 19.1 \%, 3.5 \%$, and $16.7 \%$ of patients, respectively. ENT specialists prescribed the medications to $34.3 \%, 7.8 \%, 12.4 \%, 2.2 \%$, and $13.4 \%$ of patients, respectively.

The prescription frequencies of topical corticosteroids, systemic corticosteroids, antibiotics, antihistamines, and local decongestants in patients coded as having 'nasal polyps' (ICD-10: J33) are shown in Figure 4. GP prescribed these medications to $27.3 \%$, $6.8 \%, 0.4 \%, 5.4 \%$, and $8.9 \%$ of patients, respectively. ENT specialists prescribed the medications to $71.2 \%, 23.9 \%, 1.5 \%, 3.1 \%$, and $11.5 \%$ of patients, respectively. 


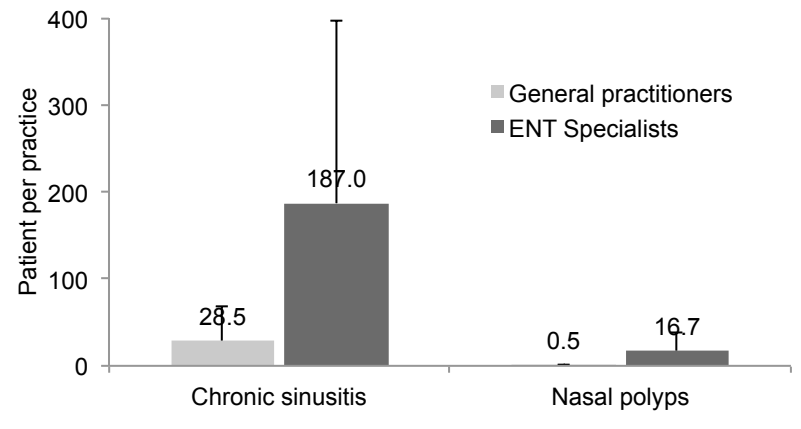

Figure 2. Patients with chronic sinus diseases per German practice.

\section{Discussion}

Other authors have reported low prevalence of corticosteroid usage. Philpott et. al found that ICS were administered in $12.1 \%$ of patients with CRSsNP and in $18.7 \%$ with CRSwNP in the UK ${ }^{(11)}$. Systemic steroids were taken by $2.89 \%$ of patients with CRSsNP and by $1.84 \%$ with CRSwNP. In the study in question, patients were treated by otolaryngologists and, according to the authors, diagnoses were made based on international guidelines. Compared to the UK study, the results of which were based on patient questionnaires, data analyzed as part of a Canadian study were retrieved from a regional administrative database of the Canadian health system ${ }^{(10)}$. It is interesting to note that, although the method of data acquisition was different, ICS utilization was similarly low at $20.1 \%$. No differentiation between CRSsNP and CRSwNP was made in this study. Furthermore, only patients who had not undergone sinus surgery were included in the study, thus all patients included in the study received presurgical treatment.

Rudmik et al. hypothesized that the majority of patients included in their study were treated by general practitioners (GP) due to the exclusion criterion of previous sinus surgery ${ }^{(10)}$. In the investigation reported by Philpott et al., otolaryngology specialists diagnosed and treated the condition ${ }^{(11)}$. Authors assumed that since patients included in the study had been referred to the specialists, CRS patients treated by GP might have responded better to ICS and were therefore not referred to the otolaryngologist as a rule. In addition, data acquisition methods varied between both studies; that is, administrative database versus questionnaire. Interestingly, the percentage of ICS usage was still indicated as similar.

Investigations with similar methodical tools as the ones used in the present study were performed in the U.S. Data from the National Ambulatory Medical Care Survey which were based on ICD-9 coding were analyzed. ICS were prescribed to $9.7 \%$ of CRS patients in GP practices and in $17.5 \%$ in otolaryngology practices ${ }^{(15)}$. ICS usage was higher for CRSwNP patients at $57.1 \%$ in GP practices and $36.3 \%$ in ENT practices ${ }^{(13)}$.

However, much higher prescription rates of ICS were also repor-
Table 1. Spread of chronic sinusitis patients in German practices (referring to Figure 2).

\begin{tabular}{|lcccc|} 
& GP & GP & ENT & ENT \\
\hline Mean & J32 & J33 & J32 & J33 \\
\hline SD & 28.5 & 0.5 & 187 & 16.7 \\
\hline Min & 39.4 & 1.3 & 209.2 & 21 \\
\hline Median & 0 & 0 & 2 & 1 \\
\hline Max & 16 & 0 & 128,5 & 11 \\
\hline IQR & 381 & 16 & 1225 & 174 \\
\hline
\end{tabular}

Data show number of patients, GP: General practitioners, ENT: ENT specialists, IQR: Interquartile range.

ted in CRS patients. Indeed, $88.6 \%$ of Dutch GPs prescribed ICS to CRS patients ${ }^{(16)}$.

In the present study, local and systemic corticosteroid usage differed between general practitioners and otolaryngologists. CRS patients seen in GP practices received treatment with ICS with similar frequency (12.3\%) as reported by other authors ${ }^{(10,11)}$. ICS were prescribed more commonly in patients with CRSwNP $(27.3 \%)$, which was even more frequently than in patients treated by otolaryngologists in the UK ${ }^{(11)}$. By comparison, the usage of ICS in German otolaryngology practices was higher than in previously reported studies from Canada and the UK ${ }^{(10,11)}$ (34.3\% in CRS patients and 71.2\% in CRSwNP patients). Differences between GPs and otolaryngologists might be attributed to patient subpopulations with more severe sinusitis disease which were referred to ENT specialists. Taking this factor into consideration, ICS usage appears to be higher in otolaryngology practices in Germany than in practices in other countries examined in previous studies ${ }^{(10,11)}$. However, ICD coding in otolaryngology practices in Germany does not distinguish whether patients did or did not undergo sinus surgery. Since operative treatment of CRS is performed by otolaryngologists, patients with sinus disease resistant to medical treatment and therefore requiring surgical intervention must have also been included, resulting in an increased percentage of patients with ICS prescriptions. Therefore, comparisons with data from other studies must be considered with caution, since Rudmik et al. only included patients without past surgical history of the sinuses ${ }^{(10)}$. Utilization of oral corticosteroids was low among CRS patients in GP (4.1\%) and in ENT (7.8\%) practices in Germany. Similar results were found in the U.S. $\left(2.3 \%\right.$ and $6.6 \%$ respectively) ${ }^{(12)}$. Systemic steroid usage was also low in the UK at $2.89 \%$ (CRS) and $1.84 \%$ (CRSwNP) ${ }^{(11)}$. Treatment of patients with CRSwNP in the U.S. included oral corticosteroids with a higher prescription rate of $28.8 \%$ in non-otolaryngology practices and of $25.9 \%$ in 


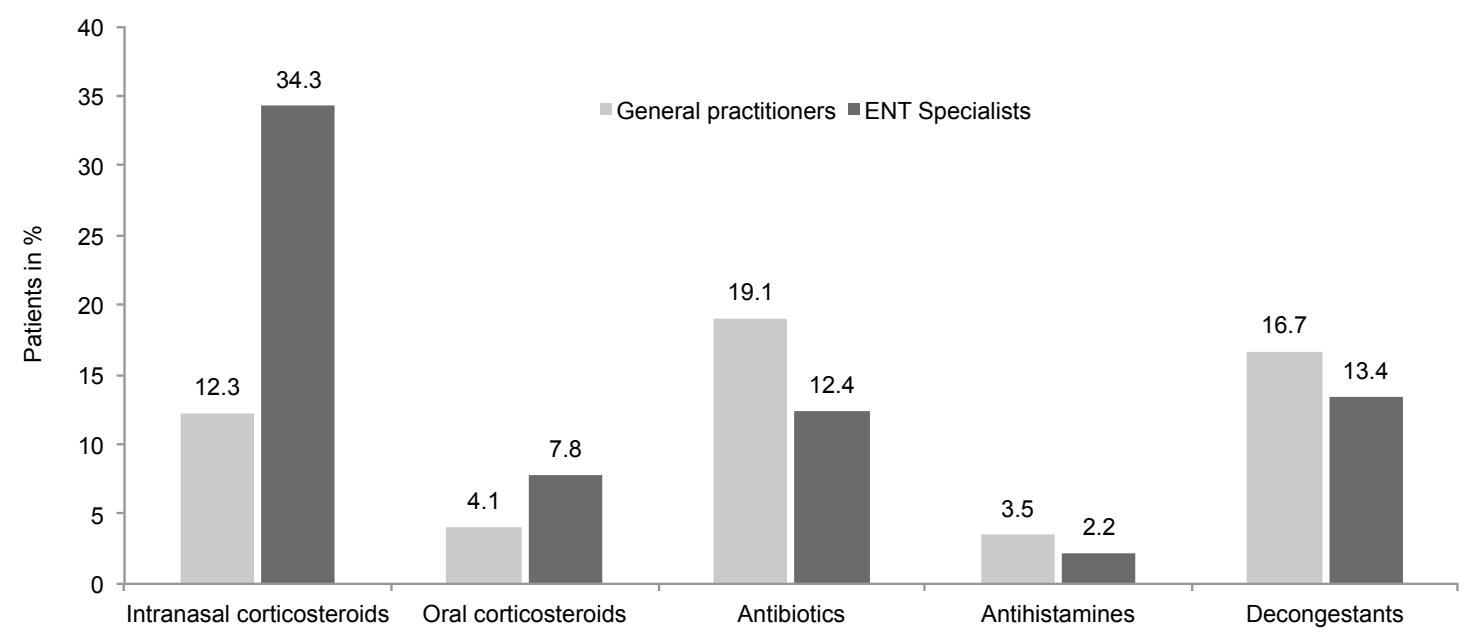

Figure 3. Medication classes prescribed to patients coded as having 'chronic sinusitis' (ICD-10: J32) in German practices.

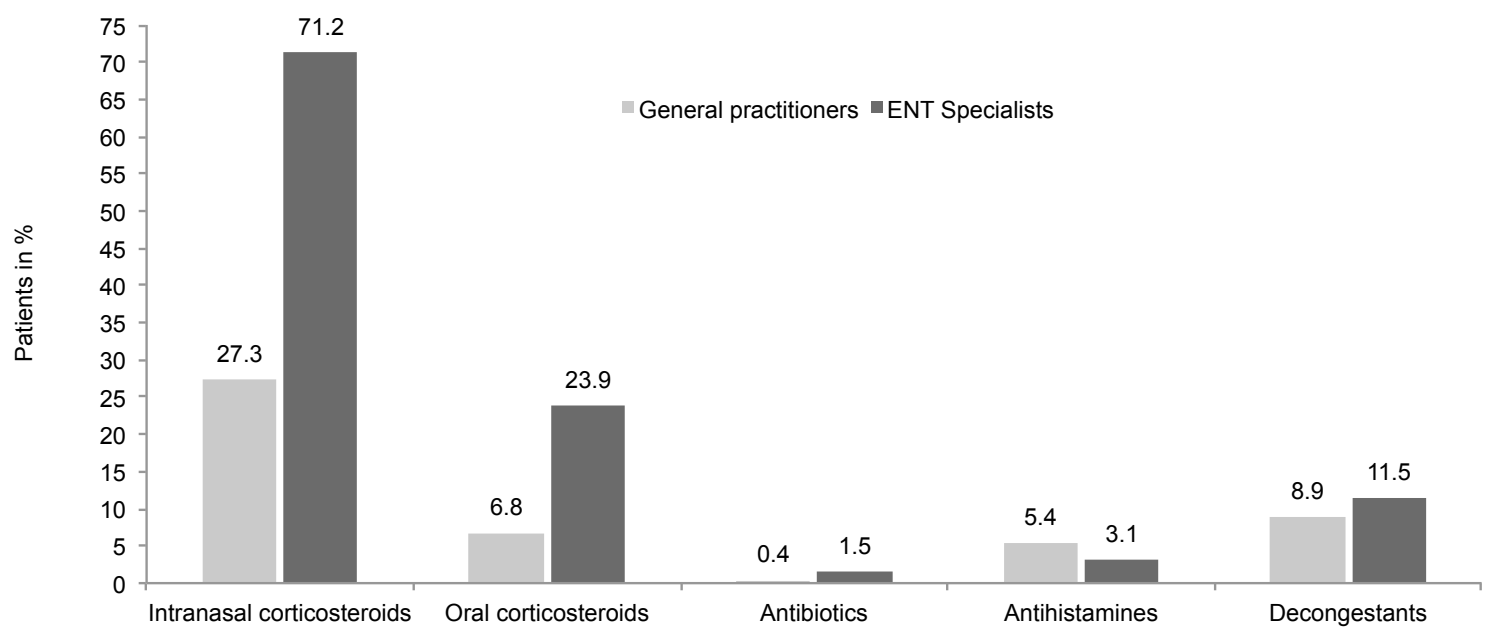

Figure 4. Medication classes prescribed to patients coded as having 'nasal polyps' (ICD-10: J33) in German practices.

ENT practices ${ }^{(17)}$. Systemic corticosteroid usage for treatment of CRSwNP was low in German GP practices (6.8\%) compared to U.S. practices but comparable to the U.S. in German otolaryngology practices (23.9\%). The difference between medical specialties may be ascribed to the referral of patients with extensive refractory sinus disease to the ENT-physicians for further treatment.

$19.1 \%$ of CRS patients were prescribed antibiotics by general practitioners in Germany, and $12.4 \%$ of CRS patients were prescribed antibiotics by otolaryngologists in Germany. Compared to data from the UK (CRSsNP: $2.89 \%$, CRSwNP: $1.54 \%)^{(11)}$, the rate of antibiotic prescriptions was higher, whereas antibiotic usage in the U.S. was even more prevalent (GP practices: $53.3 \%$, ENT practices: $27.4 \%)^{(12)}$. These figures show that antibiotics remain a commonly prescribed medication for CRS in the German ambulatory setting. By contrast, antibiotic usage in patients with CRSwNP was very low in Germany (GP practices: $0.4 \%$, ENT practices: $1.5 \%)$. However, in the US, the rate of antibiotic prescripti- ons was high among CRSwNP patients in both otolaryngology practices (22.2\%) and GP practices (32.9\%) ${ }^{(17)}$.

National differences in the usage of antihistamines were also evident. Whereas the antihistamine prescription rate in Germany was low but not negligible in CRS patients (GP practices: 3.5\%, ENT practices: $2.2 \%$ ), data from the UK showed a higher rate of antihistamine usage (8.32\%) ${ }^{(11)}$, and similar results were also reported in the U.S. $(8.7 \%)^{(15)}$. Antihistamines tend to be prescribed more frequently to CRSwNP patients. In Germany, the prescription rate was $5.4 \%$ in GP practices and $3.1 \%$ in otolaryngology practices. More frequent usage of antihistamines in CRSwNP was also seen in the UK (9.83\%) ${ }^{(11)}$ and in the U.S. (GP practices: $24.2 \%$, ENT practices: $15.3 \%)^{(17)}$. Although antihistamines are not recommended in the EPOS guidelines, they are still found in the medication regime of CRS and CRSwNP patients in Germany. However, the data analyzed in the present study does not show whether patients suffered from comorbid allergic diseases which demanded anti-allergic treatment. 
One limitation of the current study is the definition of CRS diagnosis. Since patient data are selected on the basis of ICD coding performed by the physicians in the practice, it is not known which criteria were used to define CRS. It is also not apparent whether the diagnostic criteria applied differed between practices and medical specialties, namely between general practitioners and otolaryngologists. Moreover, it is not clear whether diagnoses met the standards of international guidelines, such as the EPOS guidelines. ICD coding differentiates between J32 for "chronic sinusitis" and J33 for "nasal polyp." The fact that ICD coding cannot be regarded as equal to definitions of CRSsNP and CRSwNP as stated by the EPOS guidelines must be taken into account ${ }^{(4)}$. Aside from diagnostic criteria, the quality of ICD coding must also be addressed, since there is no control in terms of whether coding performed in the practices actually matched the patients' diseases. It cannot be ruled out that, in the course of the busy clinical routine, CRSwNP cases might also be coded as J32 ("chronic sinusitis"). Fortunately, no repercussions or sanctions for misleading coding are to be expected. However, no control system exists to verify the accuracy of ICD code assignments to patient cases. Furthermore, as has been shown in the Netherlands, acute rhinosinusitis (ARS) patients may be incorrectly coded as J32, instead of J01, which is the code for 'acute sinusitis' ${ }^{\prime}{ }^{16)}$. Dutch investigations showed that GPs differentiate between ARS and CRS, but they only use the correct coding in about half the cases. Similar miscoding cannot be ruled out in German GP practices and might occur in ENT practices as well. Such incorrect coding might explain the high percentage of antibiotic (GP: 19.1\%, ENT: 12.4\%) and decongestant (GP: 16.7\%, ENT: $13.4 \%$ ) prescriptions given to patients coded J32 (Figure 2), as well as their usage in the acute treatment of acute exacerbations of CRS. As long as the incidence of incorrect coding remains this high, it must be taken into account when interpreting the data. Another limitation of the current study is the analysis of prescribed medications by ATC classes. Although the type of medication class could be studied, the exact medication within the ATC class was not specified. Corticosteroid applications were differentiated into oral intake and local application, but intramuscular injections were not registered and could not be analyzed. Furthermore, the data do not show an accurate percentage measurement of patients who received a defined specific medical treatment in each practice. In many GP practices, only few patients (one or two) with nasal polyps were seen. As a consequence, practices whose physicians treat even a few patients would have a prescription rate of $100 \%$, whereas practices in which a few patients do not receive treatment would have a $0 \%$ prescription rate.

These aforementioned factors must be considered when interpreting the available data. Be that as it may, the advantage of the current study is the large population-based analysis representing prescription frequencies in Germany.
Guidelines in the UK require application of ICS for at least three months prior to surgical treatment and referral to an otolaryngologist ${ }^{(18)}$. Topical corticosteroid usage in German GP practices is still low and thus similar to other countries. Although ICS utilization in German otolaryngology practices is higher in CRS patients, even when compared to other countries, it is debatable whether a prescription rate of $34.3 \%$ is adequate. It has been previously discussed that the ICS prescription rate ought to be higher than studies have found it to be ${ }^{(10,11)}$, the reasoning behind this being that this would improve initial medical treatment.

ICS currently represents the only registered medication for patients with CRSwNP. EPOS guidelines recommend the application of ICS in patients with CRSwNP, since its beneficial effects have been demonstrated in many studies ${ }^{(4)}$. ICS improves symptom scores as well as nasal breathing. Furthermore, it can reduce the size of nasal polyps. Accordingly, ICS should have been prescribed in CRSwNP before moving on to other treatment options. In patients with CRSwNP who are refractory to medication, sinus surgery is currently the next therapeutic option in common otolaryngology practices. The removal of nasal polyps from the nasal and sinus cavities can improve a patient's symptoms, and it has been shown that preoperative corticosteroid administration significantly reduces intraoperative bleeding and operation time, thus leading to improved surgical field visibility which is indispensable for the sinus surgeon ${ }^{(19)}$. The preoperative effects of corticosteroids on the surgical conditions were observed after topical ${ }^{(20)}$ and oral ${ }^{(21)}$ administration. Therefore, all patients with CRSwNP who are scheduled for sinus surgery should be treated with topical corticosteroids before the operation to ensure the most favorable circumstances for a successful postoperative outcome. However, current data indicate that about $30 \%$ of CRSwNP patients treated in otolaryngology practices did not receive ICS (Figure 3). Compliance of therapy regimes with existing guidelines such as the EPOS guidelines ${ }^{(4)}$ must be subject to evaluation and appropriate reinforcement in ENT practices in Germany, and even more so in the UK.

One reason for limited ICS utilization may be the widespread usage of phytotherapy in Germany. Although clear beneficial evidence for herbal medicine in CRS is limited, phytotherapy plays a major role in non-surgical treatments as well as in GP practices and otolaryngology practices. Subsets of patients are reluctant to initiate treatment with corticosteroids or antibiotics and prefer drugs with herbal components, especially in the case of CRS. If the subjective restraint or impairment of quality of life is not substantial enough, patients may not be willing to take ICS as long as alternative preparations exist. This factor may have an additional influence on the utilization rate of corticosteroids.

However, an important fact needs to be considered. Certain ICS, antihistamines, and local decongestants are prescription- 
free medications. In Germany, certain medical products used for nasal treatment and containing budesonide or beclomethasone can be acquired as 'over-the-counter' medications. The same holds true for certain systemic antihistamines containing loratadine or cetirizine and for nasal decongestants containing xylometazoline. Therefore, the total number of patients who use the medication groups examined in this study is unknown. The data used was related only to physicians' prescription behavior, and thus considered only the medication usage attributed to physicians' orders, assuming patients followed their doctors' advice. However, a large proportion of 'over-the-counter' medication purchases are based on physicians' advice. In practice, physicians issue what is known in Germany as a'green prescription' for prescription-free medications, which the patients can purchase from pharmacies without requiring a prescription. Thus, the 'green prescription' is not an actual prescription, but more of a therapeutic recommendation for medications that are not covered by the patients' medical insurance and that need to be paid by the patients themselves. A comprehensive analysis of medication usage in CRS patients in Germany must also examine the usage of 'over-the-counter' medications, in addition to prescription medications. Such investigations have to evaluate data on medication sales in German pharmacies. Although an analysis of 'over-the-counter' medication use by CRS patients is lacking in the present study, cautious assumptions can be drawn based on data regarding prescribed medications. Since the present study shows various tendencies in the prescription behavior of physicians relating to certain medication classes, similar tendencies may also manifest when it comes to their advice on prescription-free medications. One of the main reasons physicians issue a 'green prescription' is that there is an equiva- lent 'over-the-counter' medication that is in the same medication class as the prescription drug, which means physicians do not need to stretch the limited budget for medical prescriptions that has been assigned by the health care system. Furthermore, a large number of patients can be assumed to visit physicians' practices before taken 'over-the-counter' medications, since German patients are likely to seek professional medical advice and care before taking medications, especially before using corticosteroids.

\section{Conclusion}

Topical corticosteroids usage in CRS patients in GP practices in Germany is as low as in other Western countries. Increased usage of topical corticosteroids in CRSwNP should be encouraged in GP and ENT practices. Compliance of treatment regimes in German practices with existing therapy guidelines needs to be evaluated.

\section{Authorship contribution}

JJHP contributed to the analysis and interpretation of the data, drafted the manuscript and gave the final approval of the version to be published. DUS, CB, SD contributed substantially to the interpretation of the data and gave the final approval of the version to be published. KK contributed substantially to the conception, design, and interpretation of the data, revised the manuscript critically for important content and gave the final approval of the version to be published.

\section{Conflict of interest}

None.

\section{References}

1. Hastan D, Fokkens WJ, Bachert C, Newson RB, Bislimovska J, Bockelbrink A, et al. Chronic rhinosinusitis in Europe-an underestimated disease. A GA²LEN study. Allergy. 2011 Sep;66(9):1216-23.

2. Peters AT, Spector S, Hsu J, Hamilos DL, Baroody FM, Chandra RK, et al. Diagnosis and management of rhinosinusitis: a practice parameter update. Ann Allergy Asthma Immunol. 2014 Oct;113(4):347-85

3. Lal D, Hwang PH. Oral corticosteroid therapy in chronic rhinosinusitis without polyposis: a systematic review. Int Forum Allergy Rhinol. 2011 Mar-Apr;1(2):136-43.

4. Fokkens WJ, Lund VJ, Mullol J, Bachert C, Alobid I, Baroody F, etl al. European Position Paper on Rhinosinusitis and Nasal Polyps 2012. Rhinol Suppl. 2012 Mar;23:3 p preceding table of contents, 1-298.

5. Desrosiers M, Evans GA, Keith PK, Wright ED, Kaplan A, Bouchard J, et al. Canadian clinical practice guidelines for acute and chronic rhinosinusitis. Allergy Asthma Clin Immunol. 2011 Feb 10;7(1):2.

6. Rosenfeld RM, Piccirillo JF, Chandrasekhar SS, Brook I, Ashok Kumar K, Kramper M, et al. Clinical practice guideline (update): adult sinusitis. Otolaryngol Head Neck Surg. 2015 Apr;152(2 Suppl):S1-S39.

7. Wallwork B, Coman W, Mackay-Sim A, Greiff L, Cervin A. A double-blind, randomized, placebo-controlled trial of macrolide in the treatment of chronic rhinosinusitis. Laryngoscope. 2006 Feb;116(2):189-93.

8. Videler WJ, van Hee K, Reinartz SM, Georgalas C, van der Meulen FW, Fokkens WJ. Long-term low-dose antibiotics in recalcitrant chronic rhinosinusitis: a retrospective analysis. Rhinology. 2012 Mar;50(1):4555.

9. Haye R, Aanesen JP, Burtin B, Donnelly F, Duby $C$. The effect of cetirizine on symptoms and signs of nasal polyposis. J
Laryngol Otol. 1998 Nov;112(11):1042-6.

10. Rudmik L, Xu Y, Liu M, Bird C, Kukec E, Quan H. Utilization Patterns of Topical Intranasal Steroid Therapy for Chronic Rhinosinusitis: A Canadian Population-Based Analysis. JAMA Otolaryngol Head Neck Surg. 2016 Nov 1;142(11):1056-1062.

11. Philpott C, Erskine S, Smith R, Hopkins C, Kara N, Farboud A, et al. Current use of baseline medical treatment in chronic rhinosinusitis: Data from the National Chronic Rhinosinusitis Epidemiology Study (CRES). Clin Otolaryngol. 2017 Oct 20.

12. Becher H, Kostev K, Schröder-Bernhardi D. Validity and representativeness of the "Disease Analyzer" patient database for use in pharmacoepidemiology and pharmacoeconomy. Int J Clin Pharmacol Ther. 2009 Oct;47(10):617-26.

13. Seidel DU, Jacob L, Kostev K, Sesterhenn AM. Risk factors for epistaxis in patients followed in general practices in Germany. 
Rhinology. 2017 Dec 1;55(4):312-318..

14. Seidel DU, Sesterhenn AM, Kostev K Seasonal Variation of Epistaxis in Germany. J Craniofac Surg. 2018 Jun;29(4):e365-e367.

15. Lee LN, Bhattacharyya N. Regional and specialty variations in the treatment of chronic rhinosinusitis. Laryngoscope. 2011 May;121(5):1092-7.

16. Hoffmans R, Schermer T, van Weel C, Fokkens W. Management of rhinosinusitis in Dutch general practice. Prim Care Respir J. 2011 Mar;20(1):64-70.

17. Bhattacharyya N, Kepnes LJ. Medications prescribed at ambulatory visits for nasa polyposis. Am J Rhinol Allergy. 2013 NovDec;27(6):479-81.

18. Hopkins C, Philpott C, Carrie S, et al
Commissioning guide: rhinosinusitis. London, UK: ENT UK/Royal College of Surgeons of England; 2016.

19. Hwang SH, Seo JH, Joo YH, Kang JM. Does the Preoperative Administration of Steroids Reduce Intraoperative Bleeding during Endoscopic Surgery of Nasal Polyps? Otolaryngol Head Neck Surg. 2016 Dec;155(6):949-955

20. Albu S, Gocea A, Mitre I. Preoperative treatment with topical corticoids and bleeding during primary endoscopic sinus surgery. Otolaryngol Head Neck Surg. 2010 Oct;143(4):573-8.

21. Ecevit MC, Erdag TK, Dogan E, Sutay S Effect of steroids for nasal polyposis surgery: A placebo-controlled, randomized, double-blind study. Laryngoscope. 2015 Sep;125(9):2041-5.

Prof. Dr. rer. med. Karel Kostev

Epidemiology, IQVIA

Darmstädter Landstraße 108 60598 Frankfurt am Main Germany

Tel: +49-(0)69-66 04-4878

E-mail: kkostev@de.imshealth.com

\section{ADVERTISEMENT}

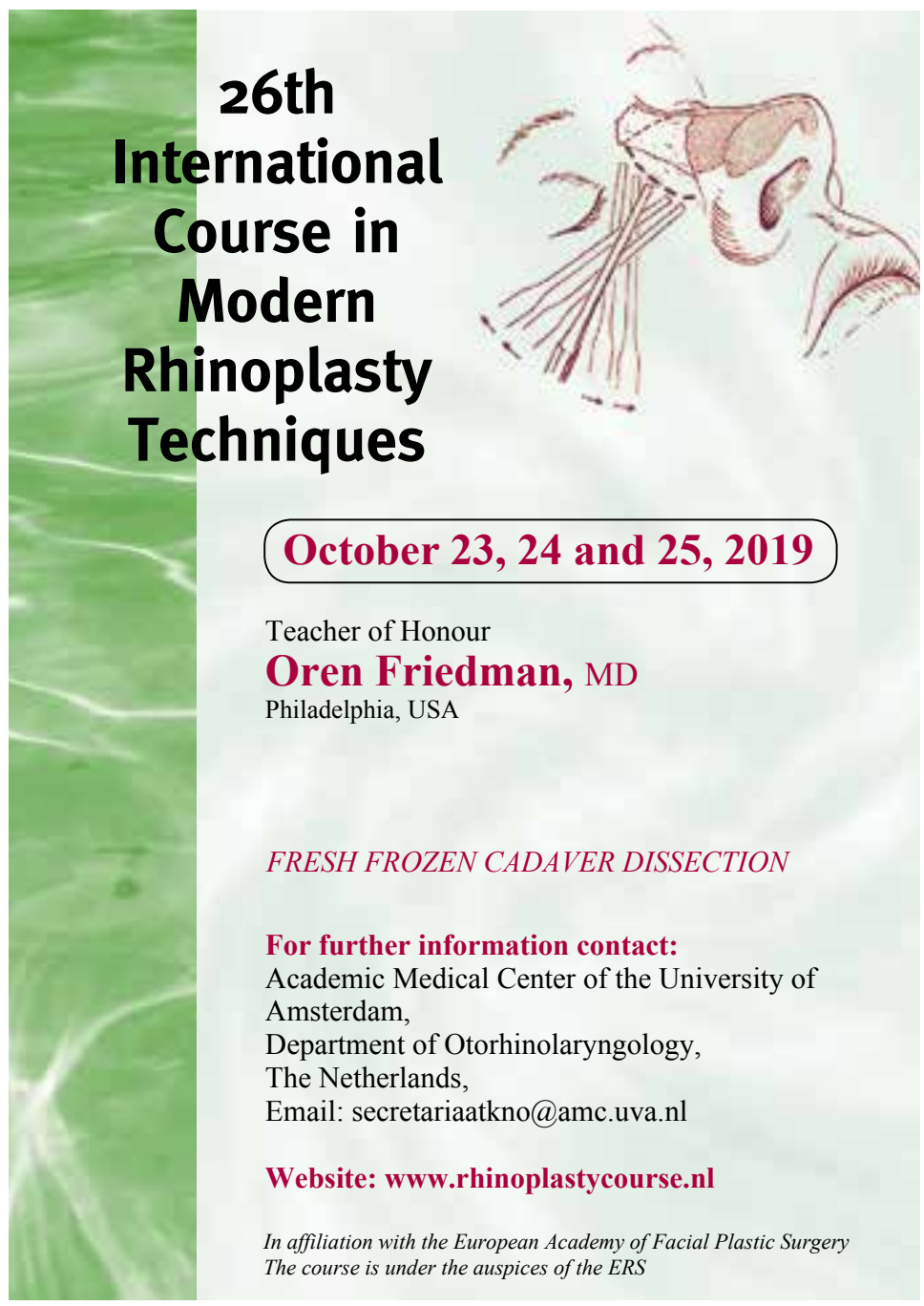

